\title{
Experimentelle Untersuchung der radialen Verformbarkeit von Stents
}

\author{
Schrader S.C., Beyar R.*, Kühler M., Kraft M., Boenick U.
}

*The Heart System Research Center, Technion - Israel Institute of Technology, Haifa, Israel
Institut für Mikrotechnik und Medizintechnik, Technische Universität Berlin

\section{EINLEITUNG}

Infolge der mechanischen Unterstützung der Gefäßwand nach Stentimplantation kann eine akute Durchmesservergrößerung gegenüber konventioneller Ballonangioplastie (PTCA) erreicht [1] und die Restenoserate gesenkt werden [2]. Eine ausreichende Widerstandsfähigkeit gegenüber äußeren Kräften ist eine Grundvoraussetzung für optimale Ergebnisse in der Stentapplikation.

Aufgrund der Vielzahl der verwendeten Materialien und Gestaltungsprinzipien bestehen große Unterschiede in dem mechanischen Verhalten der kommerziell verfugbaren Stents. Die Kenntnis dieser Verhaltensweisen stellt eine essentielle Grundlage einer an die Läsion angepaßten Stentauswahl dar. Obwohl bisher verschiedene Studien das Stentverhalten analysierten, fehlt es aufgrund unterschiedlicher Prüfverfahren an einer Vergleichbarkeit der Ergebnisse. Ziel dieses Beitrages ist die parallele Untersuchung von acht aktuellen Stenttypen. Zu diesem Zweck wurde eine Ultraschall-Meßvorrichtung zur standardisierten, quantitativen Evaluation der kompressiv-mechanischen Eigenschaften von Koronarstents entwickelt.

Mit Hilfe dieser Vorrichtung konnte darüberhinaus der Einfluß der Stentimplantation auf das radiale Dehnungsverhalten der Arterienwand untersucht werden.

\section{VERSUCHSAUFBAU UND -DURCHFÜHRUNG}

Getestet wurden acht koronare Stents: beStent (BES15), flat-wired Cardiocoil (FCR-3.0-20), Crown Stent (CR3040), Micro Stent II (RE3018), NIR Stent (9-32), articulated Palmaz-Schatz Stent (PS153-3), spirally articulated Palmaz-Schatz Stent (PS2030C) und Wiktor Stent (6575).

Es erfolgte eine Implantation der Stents in ein dünnwandiges Latexgefäß, welches zwischen den Wänden einer Druckkammer angebracht war. Durch kontinuierliche Erhöhung des hydrostatischen Druckes in der mit Wasser gefülten Kammer konnte die Kompression der Stents erreicht werden. Während der Kompression wurde der Druck mit einem Druckumformer (Modell 242PC 150G, Honeywell, USA), der Stentdurchmesser mit einem Sonomikrometer (System 6, Modell 201, Triton Technology, USA) gemessen. Die beiden Piezokristalle, zwischen denen das Sonomikrometer durch Transmission von Ultraschallimpulsen die Abstandsmessung vornimmt, wurden gegenüberliegend auf der Außenseite des Gefäßmodelles, mittig über dem Stent angebracht.

Die Verformung $\varepsilon$ wurde als relative Durchmesserverringerung infolge des zunehmenden Druckes:

$$
\varepsilon=\Delta \mathrm{d} / \mathrm{d} \text {. }
$$

( $\varepsilon$ : Verformung; $\Delta d$ : Durchmesserverringerung; d: initialer Durchmesser) gemessen. Mit Hilfe der erfaßten Druck-Verformungs-Diagramme waren der Kollabierdruck (Druck, bei dem der Stent abrupt in sich zusammenfällt) und die radiale Steifigkeit (Steigung der Kennlinie im unteren elastischen Bereich) bestimmbar. Die ballonexpandierbaren Stents wurden jeweils einmal nach einer Expansion auf $3 \mathrm{~mm}$ bzw. $4 \mathrm{~mm}$ getestet. Einzig der selbstexpandierende Cardiocoil konnte aufgrund seiner Eigenelastizität lediglich auf seinen Nenndurchmesser entfaltet werden.

Um den EinfluB der Stentimplantation auf das radiale Dehnungsverhalten des Gefäßes zu untersuchen, wurde in der Kammer ein Unterdruck erzeugt, welcher einem intraluminalen Druckanstieg gleichkommt.

\section{ERGEBNISSE I (KOMPRESSIONSVERHALTEN)}

In Abb. 1 sind die Kennlinien der getesteten Stents nach einer Expansion auf $3 \mathrm{~mm}$ dargestellt. Die Kollabierdruck- und Steifigkeitswerte, die sich aus dieser und aus der Versuchsreihe nach einer $4 \mathrm{~mm}$ Expansion ergeben sind in Abb. 2 zusammengefaßt. Die getesteten Stents konnten nach ihren Verhaltensweisen in drei Gruppen unterschieden werden:

(1) Der Durchmesser der netzartig, geschlitzt tubulären Stents (beStent, Crown, NIR und Palmaz-Schatz Stent) nahm mit steigendem Druck kontinuierlich ab, bis der maximale Druck überschritten wurde und der Stent spontan zu einem flachen, nicht passierbarem Metallgeflecht kollabierte. Infolge der unterschiedlichen

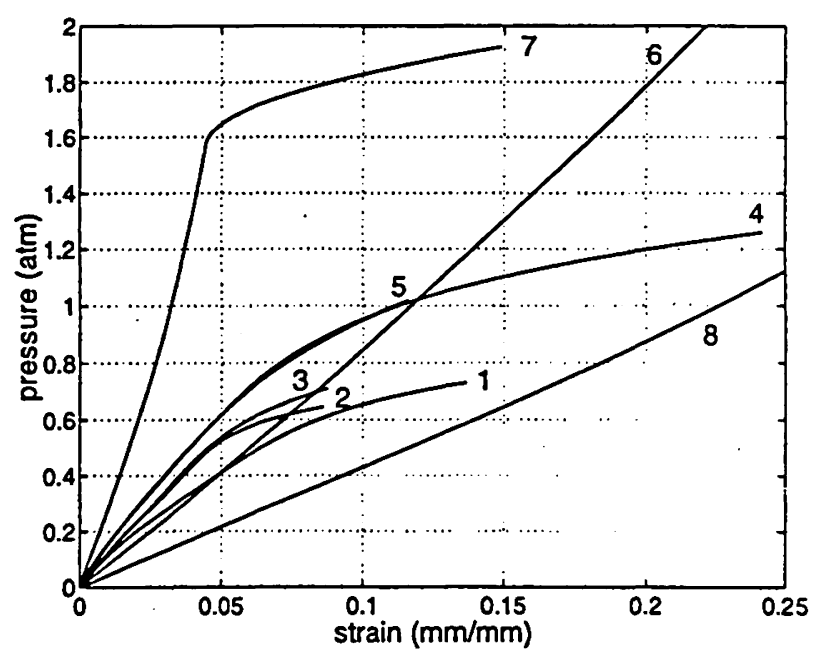

Abb. 1: Druck-Verformungverhalten der getesteten Stents nach Aufweitung auf $3 \mathrm{~mm}$. (1) beStent, (2) Palmaz-Schatz, (3) Crown, (4) spirally art. PS, (5) NIR. (6) Micro Stent II, (7) Cardiocoil, (8) Wiktor Stent. 


\begin{tabular}{|c|c|c|}
\hline Stenttyp & $\begin{array}{l}\text { Kollabierdruck } \\
\text { [atm] } \\
3 \mathrm{~mm} / 4 \mathrm{~mm}\end{array}$ & $\begin{array}{l}\text { Radiale Steifig- } \\
\text { keit }\left[\mathrm{N} / \mathrm{mm}^{2}\right] \\
3 \mathrm{~mm} / 4 \mathrm{~mm}\end{array}$ \\
\hline BeStent & $0.70 / 0.49$ & $1.0 / 0.9$ \\
\hline Paimaz-Schatz & $0.65 / 0.50$ & $1.1 / 0.8$ \\
\hline Crown & $0.71 / 0.50$ & $1.1 / 0.9$ \\
\hline NIR & $1.05 / 0.69$ & $1.2 / 0.9$ \\
\hline spirally art. PS & $1.26 / 0.83$ & $1.2 / 0.9$ \\
\hline Wiktor & $-{ }^{(a)} /-^{(b)}$ & $0.4 / 0.5$ \\
\hline Micro II & $-^{(\mathrm{a})} /-^{(\mathrm{a})}$ & $0.9 / 0.7$ \\
\hline Cardiocoil & $-^{(\mathrm{b})} /-^{\text {(c) }}$ & $4.1 /$ (c) $^{(c)}$ \\
\hline
\end{tabular}

Abb. 2: mechanische Kennwerte der getesteten Stents. ${ }^{(a)}$ kein abruptes Kollabieren; ${ }^{(b)}$ abhängig von initialer Spulenstellung; ${ }^{(c)}$ Expansion auf $4 \mathrm{~mm}$ nicht möglich.

Strebendicken und geometrischen Strebenanordnungen ergaben sich teilweise bemerkenswert unterschiedliche Kennwerte.

(2) Die aus serpentinförmig gewellten Einzeldrähten aufgebauten Stents (Wiktor und Micro Stent II) ließen sich beliebig komprimieren ohne abrupt zusammenzufallen. Auffällig sind geringe Steifigkeiten, insbesondere bei dem Wiktor Stent. Wurde der Wiktor Stent über seinen Nenndurchmesser (hier $3 \mathrm{~mm}$ ) hinaus dilatiert (auf $4 \mathrm{~mm}$ ), so mußte infolge der geometrischen Veränderungen ein abruptes Kollabieren bei geringen Drücken $(0.2-0.4 \mathrm{~atm}$, je nach initialer Spulenstellung) beobachtet werden.

(3) Der aus einer einzigen Spule bestehende Cardiocoil zeigte ein rein elastisches Verhalten. Bei Belastung nahmen die Spulen eine zunehmend schräge Lage ein, um schließlich spontan in eine Ebene abzukippen. Aufgrund der lockeren Stentstruktur wurden verschiedene Kollabierdrücke gemessen, die stark von der initialen Spulenstellung abhingen. Durch fehlerhafte Implantation (Schräglage der Spulen bereits vor Einleitung der Belastung) oder nicht-radiale Kraftanteile an der Gefäßwand, wie sie z.B. in exzentrischen Läsionen vorstellbar sind, verminderten den maximalen Kollabierdruck signifikant. Wurde der Kompressionsdruck wieder reduziert, so formte sich der Stent in seine ursprüngliche Lage zurück.

Expandiert man die Stents auf $4 \mathrm{~mm}$, so müssen im Vergleich zu einer $3 \mathrm{~mm}$ - Entfaltung stark reduzierte Kollabierdrücke und Steifigkeiten festgestellt werden. Diese Beobachtung kann auf die Laplace-Beziehung:

$$
\sigma=\mathrm{pr} / \mathrm{h}
$$

( $\sigma$ : Wandspannung eines Rohres; p: Druck; r: Rohrradius; h: Wandstärke) zurückgeführt werden. Theoretisch ist daher bei gegebenem Druck die Wandspannung innerhalb eines auf $3 \mathrm{~mm}$ entfalteten Stents um den Faktor 3/4 geringer als bei dem gleichen Stent nach $4 \mathrm{~mm}$ - Entfaltung. Die Kollabierdrücke und Steifigkeiten der getesteten Stents verhielten sich in guter Näherung an diese Beziehung.

\section{ERGEBNISSE II (DEHNUNGSVERHALTEN)}

Anhand der Kennlinien A und B (Abb. 3) ist erkennbar,

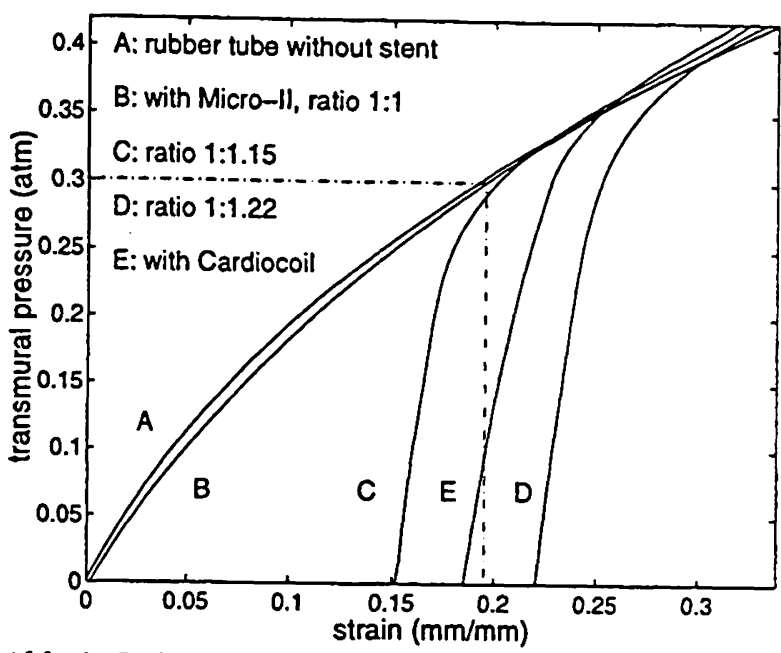

Abb. 3: Dehnungsverhalten des $3 \mathrm{~mm}$-Gefäßmodells vor und nach Implantation eines Micro Stent II (verschiedene Dilatationsgrade) und eines $4 \mathrm{~mm}$ Cardiocoil.

daß ein Stent, dessen Durchmesser dem des Gefäßmodelles angepaßt ist, keinen Einfluß auf das radiale Dehnungsverhalten des Gefäßes hat: bei steigendem Druck dehnt sich das Gefäß frei und verliert dabei den Kontakt zum Stent. Wird der Stent jedoch stärker dilatiert, so dehnt er das Gefäß (z.B. für C um 15\%) und setzt es unter eine Vorspannung. Dadurch stehen innerhalb eines Druckbereiches Stent und Gefäßwand zwar in ständigem Kontakt, jedoch tritt eine Versteifung ein, die weitgehend von der Stentsteifigkeit bestimmt wird. Die Breite des Druckbereiches hängt dabei neben dem Dilatationsgrad auch von der Elastizität des Gefäßes ab. Bei gegebenem Dilatationsgrad gilt: je steifer das Gefäß, desto großer der Druckbereich. Für das um $15 \%$ dilatierte Latexgefäß (Kennlinie $C$ ) beträgt dieser Druckbereich $0-0.3 \mathrm{~atm}$. Dies entspricht einem maximalen Blutdruck von $230 \mathrm{mmHg}$. Nach weiterer Druckerhöhung wird die Vorspannung aufgehoben und das Gefäß löst sich von dem Stent. Der Kennlinienverlauf entspricht wieder dem des stentfreien Gefäßes.

\section{DISKUSSION UND SCHLUBFOLGERUNGEN}

Die vorgestellte Meßvorrichtung ermöglicht die reproduzierbare, quantitative Bestimmung wichtiger mechanischer Kennwerte von Stents.

Innerhalb des untersuchten Stentkollektivs hat sich eine große Breite der mechanischen Kennwerte und Verhaltensweisen, die nicht nur von Gestaltungsprinip. Material und Strebendicke des Stents sondern auch von dessen Expansionsgrad abhängen, herausgestellt.

\section{LITERATURVERZEICHNIS}

[1] Haude M, Goerge G, Ge J, Baumgart D, Caspar G, Erbel R: „Treatment of in-stent restenosis. In: Siguar U, ed. Endoluminal Stenting. London:W.B. Saunders Company Lid, 1996: 357-364.

12] Fishman DL, Leon MB, Baim DS et al, for the Stent Restenosis Study Investigators: „A randomized comparison of coronary stent placement and balloon angioplasty in the treatment of coronary artery disease,.. N Engl J Med 1994; 331: 489-495. 